\title{
SMRT sequencing of the full-length transcriptome of the white-backed planthopper Sogatella furcifera
}

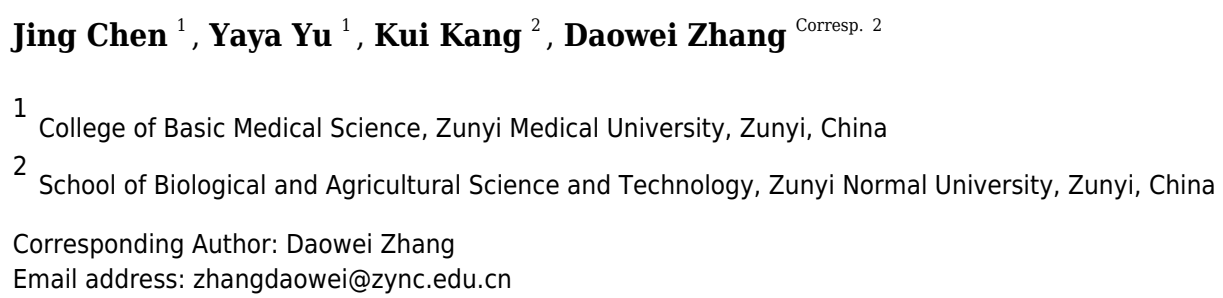

The white-backed planthopper Sogatella furcifera is an economically important rice pest distributed throughout Asia. It damages rice crops by sucking phloem sap, resulting in stunted growth and plant virus transmission. We aimed to obtain the full-length transcriptome data of $S$. furcifera using PacBio single-molecule real-time (SMRT) sequencing. Total RNA extracted from $S$. furcifera at various developmental stages (egg, larval, and adult stages) was mixed and used to generate a full-length transcriptome for SMRT sequencing. Long non-coding RNA (IncRNA) identification, full-length coding sequence prediction, full-length non-chimeric (FLNC) read detection, simple sequence repeat (SSR) analysis, transcription factor detection, and transcript functional annotation were performed. A total of 12,514,449 subreads (15.64 Gbp, clean reads) were generated, including 630,447 circular consensus sequences and 388,348 FLNC reads. Transcript cluster analysis of the FLNC reads revealed 251,109 consensus reads including 29,700 high-quality reads. Additionally, 100,360 SSRs and 121,395 coding sequences were identified using SSR analysis and ANGEL software, respectively. Furthermore, 44,324 IncRNAs were annotated using four tools and 1,288 transcription factors were identified. In total, 95,495 transcripts were functionally annotated based on searches of seven different databases. To the best of our knowledge, this is the first study of the full-length transcriptome of the white-backed planthopper obtained using SMRT sequencing. The acquired transcriptome data can facilitate further studies on the ecological and viral-host interactions of this agricultural pest. 
1 SMRT sequencing of the full-length transcriptome of

2 the white-backed planthopper Sogatella furcifera

3

4 Jing Chen ${ }^{1}$, Yaya $\mathrm{Yu}^{1}$, Kui Kang ${ }^{2}$ and Daowei Zhang ${ }^{2 *}$

$5{ }^{1}$ College of Basic Medical Science, Zunyi Medical University, Zunyi, Guizhou, People's

6 Republic of China

7 2School of Biological and Agricultural Science and Technology, Zunyi Normal University,

8 Zunyi, Guizhou, People's Republic of China

9

10

* Corresponding author

11 Daowei Zhang

12 Ping'an Avenue, Xinpu District, Zunyi City, Guizhou Province, 563006, People's Republic of 13 China

14 Email address: zhangdaowei@zync.edu.cn 


\section{Abstract}

18 The white-backed planthopper Sogatella furcifera is an economically important rice pest distributed throughout Asia. It damages rice crops by sucking phloem sap, resulting in stunted growth and plant virus transmission. We aimed to obtain the full-length transcriptome data of $S$. furcifera using PacBio single-molecule real-time (SMRT) sequencing. Total RNA extracted from $S$. furcifera at various developmental stages (egg, larval, and adult stages) was mixed and used to generate a full-length transcriptome for SMRT sequencing. Long non-coding RNA (lncRNA) identification, full-length coding sequence prediction, full-length non-chimeric (FLNC) read detection, simple sequence repeat (SSR) analysis, transcription factor detection, and transcript functional annotation were performed. A total of 12,514,449 subreads (15.64 Gbp, clean reads) were generated, including 630,447 circular consensus sequences and 388,348 FLNC reads. Transcript cluster analysis of the FLNC reads revealed 251,109 consensus reads including 29,700 high-quality reads. Additionally, 100,360 SSRs and 121,395 coding sequences were identified using SSR analysis and ANGEL software, respectively. Furthermore, 44,324 lncRNAs were annotated using four tools and 1,288 transcription factors were identified. In total, 95,495 transcripts were functionally annotated based on searches of seven different databases. To the best of our knowledge, this is the first study of the full-length transcriptome of the white-backed planthopper obtained using SMRT sequencing. The acquired transcriptome data can facilitate further studies on the ecological and viral-host interactions of this agricultural pest. 
37 Introduction

38

The white-backed planthopper (WBPH) Sogatella furcifera (Hemiptera: Delphacidae) is an economically destructive rice pest distributed throughout Asia (Ma et al. 2017; Matsumura et al. 2014). As a migratory pest, $S$. furcifera has a great ability to spread, causing widespread damage to crops (Wang et al. 2012). S. furcifera mainly affects crops in two ways, by sucking phloem sap and causing growth delay (Zhou et al. 2013); in severe cases, it causes 'hopper burn' (Lei et al. 2014; Wang et al. 2017). Furthermore, S. furcifera transmits plant viruses such as southern rice black-streaked dwarf virus (SRBSDV), rice ragged stunt virus (RRSV), and rice grassy stunt virus (RGSV) (Chen et al. 2018; He et al. 2016; Zhou et al. 2013). Currently, this pest is controlled by extensive application of chemical insecticides (Jin et al. 2017; Matsumura et al. 2014; Yang et al. 2018), but long-term use of chemical agents can lead to the development of pest resistance, causing re-infestation (Matsumura et al. 2014; Zhou et al. 2013). In addition, the overuse of pesticides can lead to environmental pollution and safety issues. Therefore, a practical approach to pest control is urgent instead of using pesticides. For example, pest control can be carried out by regulating key processes such as growth and development, immune defenses, and reproduction in pests. The target genes regulating these processes should be identified and isolated to develop new approaches to pest control. Therefore, the acquisition of long-fragment gene sequences is necessary for studies on gene functions and to identify genes that can be targeted for pest control applications.

The transcriptome can reflect the number and types of genes in a cell, revealing biochemical processes at the physiological and cellular levels (Alba et al. 2005). The next generation of highthroughput sequencing, also known as second-generation sequencing, has enabled a better understanding of various gene expression and regulation mechanisms (Bao et al. 2013). This method does not require a reference genome, and therefore, it is suitable for non-model species (Chao et al. 2018). In 2017, genome and transcriptome analyses of $S$. furcifera were performed using this method (Wang et al. 2017). De novo assembly and characterization of the S. furcifera transcriptome provided comprehensive data for gene function identification. The RNA-Seq data served as a basis for screening and analyzing candidate genes in S. furcifera for pest control. As a cogent tool for understanding gene expression and identifying splice junctions, secondgeneration sequencing has become a focus of research; however, it does not provide the fulllength sequence of all genes (Djebali et al. 2012; Nagalakshmi et al. 2008; Zhang et al. 2018). A full-length transcript is defined as the entire transcript from the $5^{\prime}$ to $3^{\prime}$ end and containing the poly-A tail. However, owing to the read-length limitation in second-generation sequencing, the transcript obtained by this method is incomplete (Wang et al. 2017). Single-molecule real-time (SMRT) sequencing, a third-generation sequencing technology developed by Pacific Biosciences 
72 (PacBio), can effectively overcome this problem (Eid et al. 2009; Korlach et al. 2010). With its 73 long read-length advantage, it can directly read reverse-transcribed full-length complementary DNA (cDNA) and provide high-quality, long, and intact transcripts (Sharon et al. 2013). The average read length is $10-15 \mathrm{~kb}$ and the maximum length is up to $70 \mathrm{~kb}$ (Stadermann et al. 2015), which helps the accurate identification of isoforms that share exons (Faist et al. 2009). Furthermore, this method can identify new genes and complement genome annotations, and contribute to the accurate analysis of fusion genes, homologous genes, and superfamily genes or alleles.

PacBio SMRT sequencing has been used for whole-transcriptome profiling of several plants, animals, and microorganisms and numerous high-confidence transcripts have been obtained (Chao et al. 2018; Jia et al. 2018; Zeng et al. 2018). Although the genome of S. furcifera has been partially sequenced (Wang et al. 2017), information about the structure and sequence of transcripts is limited and the genes have not been well annotated. In the present study, the fulllength transcriptome of $S$. furcifera was obtained by SMRT sequencing. Using the procured transcriptome data, we performed full-length sequence prediction, SSR analysis, lncRNA prediction, and gene function annotation. The transcriptome data provide numerous full-length sequences of $S$. furcifera, which will accelerate genome annotation and enhance our understanding of the genetic structure and diversity of $S$. furcifera.

\section{Materials \& Methods}

\section{Insects}

Inbred laboratory strains of $S$. furcifera originated from Zunyi Normal University of China. Continuous generations of $S$. furcifera were maintained on the susceptible rice variety Taichung Native 1 (TN1) in an artificial climatic chamber under the following stable conditions: $27 \pm 2{ }^{\circ} \mathrm{C}$, 16:8 h light/dark photoperiod, and $75 \% \pm 5 \%$ relative humidity for three years, spanning at least 30 generations. The eggs laid by $S$. furcifera within $24 \mathrm{~h}$, first to six instar larvae, and newly emerged adults starved for $24 \mathrm{~h}$ were collected and washed in precooled normal saline. Finally, $0.4 \mathrm{~g}$ of eggs, $2.0 \mathrm{~g}$ of larvae, and $5.0 \mathrm{~g}$ of adults were harvested for RNA sample preparation.

\section{RNA sample preparation}

Total RNA samples (at three developmental stages) were isolated using an RNA extraction kit (Tiangen Biotech Co., Ltd., Beijing, China). Contamination and degradation of RNA were assessed by electrophoresis on $2 \%$ agarose gels. RNA quantity was determined using a Nanodrop 2000c spectrophotometer (Thermo Fisher Scientific, Waltham, MA, USA). The concentration of 
106

107

108

109

110

111

112

113

114

115

116

117

118

119

120

121

122

123

124

125

126

127

128

129

130

131

132

133

134

135

136

137

138

139

140

141

RNA was assessed using the Qubit RNA IQ Assay Kit with the Qubit Fluorometer (Thermo Fisher Scientific). The integrity of RNA was determined using the 2100 Agilent Bioanalyzer system (Agilent Technologies, Santa Clara, CA, USA).

\section{Library construction}

The purified RNA products were sent to Novogene, Beijing, China, for SMRTbell ${ }^{\mathrm{TM}}$ library preparation and sequencing according to the manufacturer's protocol for the PacBio Sequel System (Pacific Biosciences, Menlo Park, CA, USA). Firstly, First-strand cDNA was amplified from the total RNA samples using the SMARTerTM PCR cDNA Synthesis Kit (Takara Bio USA, Inc., Mountain View, CA, USA) according to the manufacturer's protocol, and then the quality of cDNA was assessed by OD 260/280 ratio and gel electrophoresis. Finally, the double stranded cDNA was amplified by polymerase chain reaction (PCR). The BluePippin automated DNA size selection system (Sage Science, Beverly, MA, USA) was utilized to further narrow the size distribution of the final libraries ( $<4 \mathrm{~kb}$ and $\mathrm{n}>4 \mathrm{~kb}$ ). Damage repair, end repair, and SMRT dumbbell joint attachment to full-length cDNA were carried out. Finally, sequencing was performed using the PacBio Sequel single-molecule real-time sequencer (Pacific Biosciences).

\section{Preprocessing of SMRT sequencing reads}

The sequences were aligned using SMRT software (version 5.0) with the following parameters: minReadScore $=0.75$ and minLength $=200$. The quality of transcriptome completeness was assessed using Benchmarking Universal Single-Copy Orthologs (BUSCO) version 3 (Simao et al. 2015 ) with the eukaryota database. Circular consensus sequences (CCSs) were obtained from subread BAM files (parameters: max_drop_fraction, 0.8; max_length, 18000; min_length, 200; min_predicted_accuracy, 0.8; min_passes, 1; min_zscore, -999; no_polish, TRUE), with the output in CCS.BAM file format. By searching for the $5^{\prime}$ and $3^{\prime}$ end-attached adapters and the poly(A) tail, large CCSs were clustered into full-length and non-full-length reads. The full-length reads contained both $5^{\prime}$ and $3^{\prime}$ adapters and poly(A) tails. The CCSs, including these three elements and no excess copies of attached adapters within the DNA sequence, were classified as FLNC. Consensus isoforms were then obtained using Iterative Clustering for Error Correction (ICE) clustering analysis with FLNC. In addition, the consensus isoforms (specifically refers to one of the many splice variants of a gene) were polished using the non-full-length reads to identify high-quality isoforms, using Quiver for more than 99\% correction accuracy (hq_quiver_min_accuracy, 0.99; qv_trim_3p, 30; qv_trim_5p, 100; bin_size_kb, 1; bin_by_primer, false).

\section{Preprocessing of non-redundant transcripts}


142 CD-HIT (Fu et al. 2012) was used to obtain non-redundant transcripts. It is clustering and 143 redundancy software that removes redundant and similar sequences by sequence alignment 144 clustering, with the final output in non-redundant $(\mathrm{Nr})$ sequence file format. The corrected transcript sequences were non-redundant according to $99 \%$ similarity, and the distribution of length frequency before and after correction for non-redundancy was counted.

\section{CDS prediction}

The ANGEL pipeline, which is based on ANGLE (Shimizu et al. 2006), was used to identify the protein coding regions from cDNAs (https://github.com/PacificBiosciences/ANGEL). We then identified the protein sequences of $S$. furcifera, and closely related species were used for ANGEL training and prediction. Eventually, transcripts containing the 5'- and 3'-UTRs (untranslated regions) and complete CDSs were defined as full-length transcripts.

\section{Transcription factor (TF) analysis}

AnimalTFDB (Animal Transcription Factor Database v2.0, (http://bioinfo.life.hust.edu.cn/AnimalTFDB2/)) (Zhang et al. 2015) was used to analyze TFs in the $S$. furcifera transcriptome database. Because $S$. furcifera is not included in the database, the TFs were identified using hmmsearch based on the Protein Family (Pfam) search results of the TF family.

\section{IncRNA analysis}

Coding and non-coding transcripts were categorized using the following four coding potential analysis tools: Coding Potential Calculator (CPC) (Kong et al. 2007), Coding-Non-Coding Index (CNCI) (Sun et al. 2013), Predictor of long non-coding RNAs and messenger RNAs based on an improved k-mer scheme (Plek) (Li et al. 2014), and Pfam-scan (Pfam) (Finn et al. 2016). Transcripts less than $200 \mathrm{bp}$, predicted using these four tools, were removed. The CNCI tool can effectively differentiate the coding sequence of proteins and non-coding sequences free of annotations of known genes. CPC, primarily by assessing the protein-coding potential of a transcript based on biologically meaningful sequence features and identifying sequences in protein sequence databases, classifies the transcripts into coding and non-coding sequences. The NCBI eukaryotes' protein database with an e-value of $\mathrm{e}^{-10}$ was used for CPC analysis in our study. The transcript sequences predicted using Plek, CNCI, and CPC tools were used to search the Pfam-A and Pfam-B databases using hmmscan.The Pfam-A database contained high-quality domains of most known proteins, while the Pfam-B database contained more comprehensive domain families including a large number of small families for which few representative sequences are known. The transcripts that contained Pfam domains were eliminated in 
178

179

180

181

182

183

184

185

186

187

188

189

190

191

192

193

194

195

196

197

198

199

200

201

202

203

204

205

206

207

208

209

210

211

subsequent steps; default parameters were used for Pfam searches, -E 0.001 and --domE 0.001.

\section{SSR analysis}

SSRs of the transcriptome were identified using MISA 1.0 (http://pgrc.ipkgatersleben.de/misa/misa.html) (Beier et al. 2017). The unit sizes and their minimum number of repetitions were: $1-10,2-6,3-5,4-5,5-5$, and 6-5. For example, 1-10 indicates that a single nucleotide is a repeating unit and the number of repetitions is at least 10 and 2-6 indicates that a dinucleotide is a repeating unit and the minimum number of repeats is 6 .

\section{Gene function analysis}

To obtain annotation information of unigenes, various databases were utilized. Transcripts were compared against the NCBI databases Non-redundant protein sequences ( $\mathrm{Nr}$, diamond v0.8.36) (Li et al. 2002), NCBI non-redundant nucleotide sequences (Nt, ncbi-blast-2.7.1+), Swiss-Prot, Kyoto Encyclopedia of Genes and Genomes (KEGG, diamond v0.8.36) (Kanehisa et al. 2004) and Clusters of Orthologous Groups of proteins database (COG, diamond v0.8.36) (Tatusov et al. 2003) by BLASTX v2.2.31 with an E-value cut-off of $10^{-5}$. HMMER 3.1 was used to compare amino acid sequence transcripts against the Pfam database for Pfam annotation (Ventsel et al. 1973). Gene Ontology (GO) were determined based on the protein annotation results of the Pfam database (Ashburner et al. 2000).

97

\section{Results}

\section{SMRT sequencing data output}

The WBPH transcriptome was sequenced using the PacBio Sequel platform from a pooled RNA sample of $S$. furcifera obtained at different developmental stages, to accurately capture fulllength transcript isoforms (BioSample accession: SAMN12612920). RNA was isolated from pooled samples and the cDNA was classified as fractions of full-length transcripts up to $4 \mathrm{~kb}$ (without performing size selection). The analysis of transcriptome completeness with BUSCO was shown in Table 1. A total of $15.64 \mathrm{Gbp}$ sequence data in 12,514,449 PacBio subreads were obtained, with an average sequence length of 1,250 bp and N50 of 2,665 bp (Table 2, Fig. 1). To provide more accurate and reliable sequences, CCSs were generated from a single molecule sequence, and finally, 630,447 CCSs were obtained. By detecting the sequences, 421,026 CCSs were identified as full-length reads (containing $5^{\prime}$ and $3^{\prime}$ signals plus the poly(A) tails) and 388,348 were identified as FLNC reads with a mean length of 2,682 bp (Table 3, Fig. 1). The FLNC reads with highly similar sequences were clustered together into one consensus sequence 
212 using the ICE algorithm, resulting in 251,109 consensus isoforms (Table 3, Fig. 1), in 213 combination with non-full-length sequences. Eventually, using the Quiver program to cluster 214 non-full-length sequences, the resulting sequences were corrected, generating 29,700 high215 quality isoforms (HQs).

216

\section{Non-redundant transcript acquisition}

218

219

220

221

222

223

224

225

226

227

228

229

230

231

232

233

234

235

236

237

238

239

240

241

242

243

244

245

246

247

To obtain a series of non-redundant transcripts, we clustered highly similar coding sequences together using CD-HIT with an amino-acid sequence identity threshold of 99\%. All 251,109 consensus transcripts were used for the acquisition of non-redundant transcripts, and finally, 156,138 unigenes (mean length of $2994 \mathrm{bp}$ ) were obtained. The length distribution of the unigenes was counted at an interval of every 500-1000 bp. As shown in Table 4, unigenes longer than 3000 bp accounted for $46.6 \%$, whereas those shorter than 500 bp only accounted for $8.9 \%$ of the total genes. As shown in Table 5, 92.5\% of unigenes had only one isoform, while only $7.5 \%$ of unigenes had two to ten isoforms.

\section{SSR detection}

A total of 100,360 SSR sequences $(5,833,286$ bp) were obtained, with 74,401 SSRs and 25,959 SSR-containing sequences. The cumulative number of sequences with at least one SSR was 73,671 and the number of SSRs present in compound formation was 27,268. Moreover, SSRs consisting of one to six (mono-, di-, tri-, tetra-, penta-, and hexa-nucleotide) tandem repeats were identified. Mono-repeats $(46,035 ; 62.98 \%)$ were the most abundant in the WBPH unigenes, followed by tri-repeats $(21,834 ; 29.87 \%)$ and di-repeats $(4,453 ; 6.09 \%)$. The frequencies of tetra-, penta-, and hexa-repeat types were only $0.86 \%$ (632), $0.14 \%$ (99), and $0.05 \%$ (39) in the WBPH unigenes, respectively (Fig. 2).

\section{TF detection}

We analyzed TFs by comparing the transcript sequences to those in the AnimalTFDB 2.0 database, resulting in 1,288 TFs. The numbers of TFs enriched were as follows: zf-C2H2 (425), ZBTB (166), TF_bZIP (71), Homeobox (68), and HMG (60) (Fig. 3).

\section{Prediction of coding sequences}

We used verified protein sequences of this species or closely related species for ANGEL training and then ran the ANGEL prediction for the given sequences. Then, 121,395 coding sequences were generated; among them, 48,873 coding sequences containing the start and stop codons were defined as complete open reading frames (ORFs). Subsequently, the number, size, and length distributions of the $5^{\prime}$ and $3^{\prime}$ UTR regions were analyzed. In total, 8,225 transcripts were 
248

249

250

251

252

253

254

255

256

257

258

259

260

261

262

263

264

265

266

267

268

269

270

271

272

273

274

275

276

277

278

279

280

281

282

annotated to the $5^{\prime}$-UTR regions and 2,231 to the $3^{\prime}$-UTR regions. As shown in Fig 4, CDS lengths $\leq 1000 \mathrm{bp}$ accounted for $85.5 \%(103,696)$, followed by those from 1000-2000 bp $(14,625 ; 12.0 \%)$ and $>2000$ bp $(3,074 ; 2.5 \%)$ (Fig. 4).

\section{Identification of IncRNAs}

We used four tools to identity unique transcripts without protein coding potential (i.e., lncRNAs). The CNCI tool identified 110,664 lncRNAs, Pfam identified 121,199 lncRNAs, Plek identified 51,434 lncRNAs, and CPC identified 98,911 lncRNAs. In total, 44,324 lncRNA transcripts were predicted by all four methods (Fig. 5).

\section{Functional annotation of transcripts}

All 156,138 corrected transcripts were utilized to annotate according to function via searching the GO, KEGG, COG, NR, NT, Pfam, and SwissProt databases, and 95,495 transcripts (61.2\%) were annotated (Fig. 6A). Firstly, the transcripts were compared to those in the NR database (70,969 transcripts). The results showed that species with the most matching transcripts belong to Hemiptera insects including Clastoptera arizonana (11,731), Nilaparvata lugens $(6,983)$, Cuerna arida (6,190), Graphocephala atropunctata (6,052), and Homalodisca liturata $(5,485)$ (Fig. 6B). KEGG analysis showed that 60,352 transcripts were assigned to 356 KEGG pathways and clustered significantly in signal transduction, transport and catabolism, and endocrine system (Fig. 6C). The COGs functional classification of the transcripts is shown in Fig. 6D, 44,948 transcripts matched an entry in COG. Finally, the result of GO analysis showed that 37,604 transcripts were enriched in the pathways related to biological processes, cellular components, and molecular functions. Most of the genes were involved in the following "biological processes": cellular process, metabolic process, single-organism process, location, regulation of biological processes, and biological regulation. A high number of genes in "cellular components" were mainly involved in cell, cell part, membrane, organelle, and virion. The category "molecular functions" mainly consisted of transcripts involved in catalytic, binding, transporter, nucleic acid binding transcription factor, and structural molecular activities (Fig. 6E).

\section{Discussion}

The SMRT sequencing platform produces long reads that can effectively resolve the issue of completed gene structures. The strengths of PacBio RNA sequencing have been widely investigated in various species (Allen et al. 2017; Chao et al. 2018; Jia et al. 2018; Park et al. 2017; Sharon et al. 2013; Tombacz et al. 2017; Zeng et al. 2018). To date, a draft genome sequence of $S$. furcifera has been published (Wang et al. 2017), but the sequences of most full- 
283

284

285

286

287

288

289

290

291

292

293

294

295

296

297

298

299

300

301

302

303

304

305

306

307

308

309

310

311

312

313

314

315

316

317

318

length transcripts are still unknown. We mapped SMRT sequencing transcripts to the $S$. furcifera reference genome (Wang et al. 2017) using GMAP (Wu \& Watanabe 2005). The percentage of mapped transcripts occupied $64.79 \%$ and unmapped occupied $35.21 \%$ (Table S1). As we mentioned, rice virus SRBSDV, RRSV and RGSV were transmitted by S. furcifera, so there were some of the transcript sequences appear to be of viral origin in our sequencing results.

One of the advantages of SMRT sequencing is that it provides new comprehension of fulllength sequences, gene structures, and gene functions. The results of our study indicate that SMRT sequencing is useful for both genome annotation and gene function studies (Dong et al. 2015). The full-length transcriptome of S. furcifera was analyzed using PacBio SMRT sequencing and $15.64 \mathrm{Gbp}$ of clean data was obtained, including 630,447 CCSs, of which 388,348 were identified as FLNC transcripts. In addition, 251,109 consensus transcripts were obtained using transcript cluster analysis of the FLNC reads, which included 29,700 high-quality transcripts. The lengths of the FLNC transcripts were aligned to the sizes of the transcriptome library (Fig. 1B). PacBio CCSs and FLNC reads did not require assembly of short next generation sequencing (NGS) reads. Furthermore, the high competence of PacBio SMRT sequencing to generate full-length transcript sequence may well be connected with its long-read property. In our study, 156,138 unigenes with mean length of 2,994 bp were obtained from the SMRT sequencing platform, which is much better than those assembled using only Illumina sequencing. For example, Li et al obtained 51,842 unigenes with a mean length of $746 \mathrm{bp}$ ( $\mathrm{Li}$ et al. 2016), and Xu et al obtained 81,388 unigenes with a mean length of $555 \mathrm{bp}$ (Xu et al. 2012).

lncRNAs are a class of non-protein-encoding transcripts of over $200 \mathrm{nt}$ in length that are important to regulate gene expression at various levels. Currently, the roles of IncRNAs in insects, such as Drosophila melanogaster, Agasicles hygrophila, Plutella xylostella, and $N$. lugens, have been reported. Studies on D. melanogaster have shown that lncRNAs are involved in sex determination (Mulvey et al. 2014), motor behavior and climbing ability (Li et al. 2012), courtship behavior in males (Chen et al. 2011), sleep behavior (Soshnev et al. 2011), and inactivation of X staining (Deng \& Meller 2006; Smith et al. 2001). In recent years, with increasingly intense research on IncRNA functions, the RNA-Seq data of some insects are being re-explored and several insect lncRNAs have been discovered. While studies on the function of IncRNA in fruit flies and honeybees have made crucial progress, in other insects, such as agricultural pests, the study of IncRNA function is impeded by several difficulties and challenges. In our study, 44,324 lncRNAs were predicted by all four methods, and their function in $S$. furcifera requires further investigation.

Full-length transcript sequence information is crucial for genome annotation and gene function research. However, most methods used to obtain a full-length cDNA clone are time consuming, expensive, and inefficient (Bower \& Johnston 2010; Chen et al. 2016; Schmidt \&

PeerJ reviewing PDF | (2019:11:43416:3:0:NEW 6 May 2020) 
319

320

321

322

323

324

325

326

327

328

329

330

331

332

333

334

335

336

337

338

339

340

341

342

343

344

345

346

347

348

349

350

351

352

353

Mueller 1999). To date, only a few full-length cDNA sequences have been reported in $S$. furcifera. Recently, Liang et al. used second-generation sequencing technology to sequence the transcriptome of $S$. furcifera and reported that the unigene length was 200-400 bp, accounting for $41.50 \%$ of the total number of genes, whereas those longer than 4,000 bp only accounted for $3.37 \%$. Furthermore, 18,416 ORFs were generated based on the unigenes. In addition, the generated ORFs were compared with those in the Nr, Swiss-Prot, GO, COG, and KEGG databases, and 18,415 transcripts were annotated (Liang et al. 2018). In the present study, we used the PacBio SMRT sequencing platform to obtain 100,360 SSRs and 121,395 CDSs, of which 48,873 carried complete ORFs. Unigenes longer than 4000 bp comprised $26.4 \%$ of the total number of genes, whereas those shorter than 500 bp accounted for $8.9 \%$ (Table 4). Therefore, our results show that SMRT sequencing is a useful and effective tool for acquiring reliable full-length transcripts of $S$. furcifera. Using SMRT sequencing, 95,495 transcripts were annotated, which will assist future research and the identification of gene functions in $S$. furcifera.

\section{Conclusions}

Through the PacBio SMRT sequencing platform, a large collection of full-length transcripts were obtained. The number and mean length of the unigenes, as well as the number of complete ORFs from SMRT sequencing were much better than those from Illumina sequencing. The obtained transcriptome data can assist further studies on gene function in S. furcifera and help clarify the interaction of S. furcifera in the ecosystem.

\section{References}

Alba R, Payton P, Fei Z, McQuinn R, Debbie P, Martin GB, Tanksley SD, and Giovannoni JJ. 2005. Transcriptome and selected metabolite analyses reveal multiple points of ethylene control during tomato fruit development. Plant Cell 17:2954-2965. 10.1105/tpc.105.036053

Allen SL, Delaney EK, Kopp A, and Chenoweth SF. 2017. Single-Molecule Sequencing of the Drosophila serrata Genome. G3 (Bethesda) 7:781-788. 10.1534/g3.116.037598

Ashburner M, Ball CA, Blake JA, Botstein D, Butler H, Cherry JM, Davis AP, Dolinski K, Dwight SS, Eppig JT, Harris MA, Hill DP, Issel-Tarver L, Kasarskis A, Lewis S, Matese JC, Richardson JE, Ringwald M, Rubin GM, and Sherlock G. 2000. Gene ontology: tool for the unification of biology. The Gene Ontology Consortium. Nat Genet 25:25-29. 10.1038/75556

Bao YY, Qu LY, Zhao D, Chen LB, Jin HY, Xu LM, Cheng JA, and Zhang CX. 2013. The genome- and transcriptomewide analysis of innate immunity in the brown planthopper, Nilaparvata lugens. BMC Genomics 14:160. 10.1186/1471-2164-14-160

Beier S, Thiel T, Munch T, Scholz U, and Mascher M. 2017. MISA-web: a web server for microsatellite prediction. 
Bioinformatics 33:2583-2585. 10.1093/bioinformatics/btx198

Bower NI, and Johnston IA. 2010. Targeted rapid amplification of CDNA ends (T-RACE)--an improved RACE reaction through degradation of non-target sequences. Nucleic Acids Res 38:e194. 10.1093/nar/gkq816

Chao Y, Yuan J, Li S, Jia S, Han L, and Xu L. 2018. Analysis of transcripts and splice isoforms in red clover (Trifolium pratense L.) by single-molecule long-read sequencing. BMC Plant Biol 18:300. 10.1186/s12870-018-1534-8

Chen J, Zhang DW, Jin X, Xu XL, and Zeng BP. 2018. Characterization of the Akirin Gene and Its Role in the NFkappaB Signaling Pathway of Sogatella furcifera. Front Physiol 9:1411. 10.3389/fphys.2018.01411

Chen N, Wang WM, and Wang HL. 2016. An efficient full-length cDNA amplification strategy based on bioinformatics technology and multiplexed PCR methods. Sci Rep 5:19420. 10.1038/srep19420

Chen Y, Dai H, Chen S, Zhang L, and Long M. 2011. Highly tissue specific expression of Sphinx supports its male courtship related role in Drosophila melanogaster. PLoS One 6:e18853. 10.1371/journal.pone.0018853

Deng X, and Meller VH. 2006. roX RNAs are required for increased expression of X-linked genes in Drosophila melanogaster males. Genetics 174:1859-1866. 10.1534/genetics.106.064568

Djebali S, Davis CA, Merkel A, Dobin A, Lassmann T, Mortazavi A, Tanzer A, Lagarde J, Lin W, Schlesinger F, Xue C, Marinov GK, Khatun J, Williams BA, Zaleski C, Rozowsky J, Röder M, Kokocinski F, Abdelhamid RF, Alioto T, Antoshechkin I, Baer MT, Bar NS, Batut P, Bell K, Bell I, Chakrabortty S, Chen X, Chrast J, Curado J, Derrien T, Drenkow J, Dumais E, Dumais J, Duttagupta R, Falconnet E, Fastuca M, Fejes-Toth K, Ferreira P, Foissac S, Fullwood MJ, Gao H, Gonzalez D, Gordon A, Gunawardena H, Howald C, Jha S, Johnson R, Kapranov P, King B, Kingswood C, Luo OJ, Park E, Persaud K, Preall JB, Ribeca P, Risk B, Robyr D, Sammeth M, Schaffer L, See L-H, Shahab A, Skancke J, Suzuki AM, Takahashi H, Tilgner H, Trout D, Walters N, Wang H, Wrobel J, Yu Y, Ruan X, Hayashizaki Y, Harrow J, Gerstein M, Hubbard T, Reymond A, Antonarakis SE, Hannon G, Giddings MC, Ruan Y, Wold B, Carninci P, Guigó R, and Gingeras TR. 2012. Landscape of transcription in human cells. Nature 489:101. 10.1038/nature11233

https://www.nature.com/articles/nature11233\#supplementary-information

Dong L, Liu H, Zhang J, Yang S, Kong G, Chu JS, Chen N, and Wang D. 2015. Single-molecule real-time transcript sequencing facilitates common wheat genome annotation and grain transcriptome research. $B M C$ Genomics 16:1039. 10.1186/s12864-015-2257-y

Eid J, Fehr A, Gray J, Luong K, Lyle J, Otto G, Peluso P, Rank D, Baybayan P, Bettman B, Bibillo A, Bjornson K, Chaudhuri B, Christians F, Cicero R, Clark S, Dalal R, Dewinter A, Dixon J, Foquet M, Gaertner A, Hardenbol $P$, Heiner $C$, Hester $K$, Holden $D$, Kearns $G$, Kong $X$, Kuse $R$, Lacroix $Y$, Lin $S$, Lundquist $P$, Ma C, Marks $P$, Maxham M, Murphy D, Park I, Pham T, Phillips M, Roy J, Sebra R, Shen G, Sorenson J, Tomaney A, Travers K, Trulson M, Vieceli J, Wegener J, Wu D, Yang A, Zaccarin D, Zhao P, Zhong F, Korlach J, and Turner S. 2009. Real-time DNA sequencing from single polymerase molecules. Science 323:133-138. 10.1126/science.1162986

Faist F, Short S, Kneale GG, and Sharpe CR. 2009. Alternative splicing determines the interaction of SMRT isoforms with nuclear receptor-DNA complexes. Biosci Rep 29:143-149. 10.1042/BSR20080093

Finn RD, Coggill P, Eberhardt RY, Eddy SR, Mistry J, Mitchell AL, Potter SC, Punta M, Qureshi M, Sangrador-Vegas A, Salazar GA, Tate J, and Bateman A. 2016. The Pfam protein families database: towards a more sustainable future. Nucleic Acids Res 44:D279-285. 10.1093/nar/gkv1344

Fu L, Niu B, Zhu Z, Wu S, and Li W. 2012. CD-HIT: accelerated for clustering the next-generation sequencing data. Bioinformatics 28:3150-3152. 10.1093/bioinformatics/bts565 
395

396

397

398

399

400

401

402

403

404

405

406

407

408

409

410

411

412

413

414

415

416

417

418

419

420

421

422

423

424

425

426

427

428

429

430

431

432

433

434

435

He K, Lin K, Wang G, and Li F. 2016. Genome Sizes of Nine Insect Species Determined by Flow Cytometry and k-mer Analysis. Front Physiol 7:569. 10.3389/fphys.2016.00569

Jia D, Wang Y, Liu Y, Hu J, Guo Y, Gao L, and Ma R. 2018. SMRT sequencing of full-length transcriptome of flea beetle Agasicles hygrophila (Selman and Vogt). Sci Rep 8:2197. 10.1038/s41598-018-20181-y

Jin JX, Jin DC, Li WH, Cheng Y, Li FL, and Ye ZC. 2017. Monitoring Trends in Insecticide Resistance of Field Populations of Sogatella furcifera (Hemiptera: Delphacidae) in Guizhou Province, China, 2012-2015. J Econ Entomol 110:641-650. 10.1093/jee/tox027

Kanehisa M, Goto S, Kawashima S, Okuno Y, and Hattori M. 2004. The KEGG resource for deciphering the genome. Nucleic Acids Res 32:D277-280. 10.1093/nar/gkh063

Kong L, Zhang Y, Ye ZQ, Liu XQ, Zhao SQ, Wei L, and Gao G. 2007. CPC: assess the protein-coding potential of transcripts using sequence features and support vector machine. Nucleic Acids Res 35:W345-349. 10.1093/nar/gkm391

Korlach J, Bjornson KP, Chaudhuri BP, Cicero RL, Flusberg BA, Gray JJ, Holden D, Saxena R, Wegener J, and Turner SW. 2010. Real-time DNA sequencing from single polymerase molecules. Methods Enzymol 472:431-455. 10.1016/S0076-6879(10)72001-2

Lei W, Liu D, Li P, and Hou M. 2014. Interactive Effects of Southern Rice Black-Streaked Dwarf Virus Infection of Host Plant and Vector on Performance of the Vector, Sogatella furcifera (Homoptera: Delphacidae). J Econ Entomol 107:1721-1727. 10.1603/EC13569

Li A, Zhang J, and Zhou Z. 2014. PLEK: a tool for predicting long non-coding RNAs and messenger RNAs based on an improved k-mer scheme. BMC Bioinformatics 15:311. 10.1186/1471-2105-15-311

Li M, Wen S, Guo X, Bai B, Gong Z, Liu X, Wang Y, Zhou Y, Chen X, Liu L, and Chen R. 2012. The novel long noncoding RNA CRG regulates Drosophila locomotor behavior. Nucleic Acids Res 40:11714-11727. 10.1093/nar/gks943

Li W, Jaroszewski L, and Godzik A. 2002. Tolerating some redundancy significantly speeds up clustering of large protein databases. Bioinformatics 18:77-82.

Li Z, An XK, Liu YD, and Hou ML. 2016. Transcriptomic and Expression Analysis of the Salivary Glands in WhiteBacked Planthoppers, Sogatella furcifera. PLoS One 11:e0159393. 10.1371/journal.pone.0159393

Liang AW, Zhang H, Lin J, and Wang FH. 2018. De Novo Assembly and Analysis of the White-Backed Planthopper (Sogatella furcifera) Transcriptome. J Insect Sci 18. 10.1093/jisesa/iey074

Ma M, Wu Y, Peng Z, Zhao X, Zhang Y, Liao G, and Zhai B. 2017. Migration Analysis of Sogatella furcifera (Hemiptera: Delphacidae) in the Northeastern Hunan Province in June. Environ Entomol 46:757-765. 10.1093/ee/nvx092

Matsumura M, Sanada-Morimura S, Otuka A, Ohtsu R, Sakumoto S, Takeuchi H, and Satoh M. 2014. Insecticide susceptibilities in populations of two rice planthoppers, Nilaparvata lugens and Sogatella furcifera, immigrating into Japan in the period 2005-2012. Pest Manag Sci 70:615-622. 10.1002/ps.3590

Mulvey BB, Olcese U, Cabrera JR, and Horabin JI. 2014. An interactive network of long non-coding RNAs facilitates the Drosophila sex determination decision. Biochim Biophys Acta 1839:773-784. 10.1016/j.bbagrm.2014.06.007

Nagalakshmi U, Wang Z, Waern K, Shou C, Raha D, Gerstein M, and Snyder M. 2008. The transcriptional landscape of the yeast genome defined by RNA sequencing. Science 320:1344-1349. 10.1126/science.1158441

Park SG, Yoo SI, Ryu DS, Lee H, Ahn YJ, Ryu H, Ko J, and Hong CP. 2017. Long-read transcriptome data for improved

Peer) reviewing PDF | (2019:11:43416:3:0:NEW 6 May 2020) 
436

437

438

439

440

441

442

443

444

445

446

447

448

449

450

451

452

453

454

455

456

457

458

459

460

461

462

463

464

465

466

467

468

469

470

471

472

473

474

475

476

gene prediction in Lentinula edodes. Data Brief 15:454-458. 10.1016/j.dib.2017.09.052

Schmidt WM, and Mueller MW. 1999. CapSelect: a highly sensitive method for 5' CAP-dependent enrichment of full-length cDNA in PCR-mediated analysis of mRNAs. Nucleic Acids Res 27:e31. 10.1093/nar/27.21.e31

Sharon D, Tilgner H, Grubert F, and Snyder M. 2013. A single-molecule long-read survey of the human transcriptome. Nat Biotechnol 31:1009-1014. 10.1038/nbt.2705

Shimizu K, Adachi J, and Muraoka Y. 2006. ANGLE: a sequencing errors resistant program for predicting protein coding regions in unfinished cDNA. J Bioinform Comput Biol 4:649-664.

Simao FA, Waterhouse RM, Ioannidis P, Kriventseva EV, and Zdobnov EM. 2015. BUSCO: assessing genome assembly and annotation completeness with single-copy orthologs. Bioinformatics 31:3210-3212. 10.1093/bioinformatics/btv351

Smith ER, Allis CD, and Lucchesi JC. 2001. Linking global histone acetylation to the transcription enhancement of Xchromosomal genes in Drosophila males. J Biol Chem 276:31483-31486. 10.1074/jbc.C100351200

Soshnev AA, Ishimoto H, McAllister BF, Li X, Wehling MD, Kitamoto T, and Geyer PK. 2011. A conserved long noncoding RNA affects sleep behavior in Drosophila. Genetics 189:455-468. 10.1534/genetics.111.131706

Stadermann KB, Weisshaar B, and Holtgrawe D. 2015. SMRT sequencing only de novo assembly of the sugar beet (Beta vulgaris) chloroplast genome. BMC Bioinformatics 16:295. 10.1186/s12859-015-0726-6

Sun L, Luo H, Bu D, Zhao G, Yu K, Zhang C, Liu Y, Chen R, and Zhao Y. 2013. Utilizing sequence intrinsic composition to classify protein-coding and long non-coding transcripts. Nucleic Acids Res 41:e166. 10.1093/nar/gkt646

Tatusov RL, Fedorova ND, Jackson JD, Jacobs AR, Kiryutin B, Koonin EV, Krylov DM, Mazumder R, Mekhedov SL, Nikolskaya AN, Rao BS, Smirnov S, Sverdlov AV, Vasudevan S, Wolf YI, Yin JJ, and Natale DA. 2003. The COG database: an updated version includes eukaryotes. BMC Bioinformatics 4:41. 10.1186/1471-2105-441

Tombacz D, Balazs Z, Csabai Z, Moldovan N, Szucs A, Sharon D, Snyder M, and Boldogkoi Z. 2017. Characterization of the Dynamic Transcriptome of a Herpesvirus with Long-read Single Molecule Real-Time Sequencing. Sci Rep 7:43751. 10.1038/srep43751

Ventsel SV, Kriuchkov V, and Nesterenko VA. 1973. [Friction conditions in microtomes]. Med Tekh 2:30-32.

Wang L, Tang N, Gao X, Chang Z, Zhang L, Zhou G, Guo D, Zeng Z, Li W, Akinyemi IA, Yang H, and Wu Q. 2017. Genome sequence of a rice pest, the white-backed planthopper (Sogatella furcifera). Gigascience 6:1-9. 10.1093/gigascience/giw004

Wang Y, Fan HW, Huang HJ, Xue J, Wu WJ, Bao YY, Xu HJ, Zhu ZR, Cheng JA, and Zhang CX. 2012. Chitin synthase 1 gene and its two alternative splicing variants from two sap-sucking insects, Nilaparvata lugens and Laodelphax striatellus (Hemiptera: Delphacidae). Insect Biochem Mol Biol 42:637-646. 10.1016/j.ibmb.2012.04.009

Wu TD, and Watanabe CK. 2005. GMAP: a genomic mapping and alignment program for mRNA and EST sequences. Bioinformatics 21:1859-1875. 10.1093/bioinformatics/bti310

$\mathrm{Xu} Y$, Zhou W, Zhou Y, Wu J, and Zhou X. 2012. Transcriptome and comparative gene expression analysis of Sogatella furcifera (Horvath) in response to southern rice black-streaked dwarf virus. PLoS One 7:e36238. 10.1371/journal.pone.0036238

Yang YX, Yu N, Zhang JH, Zhang YX, and Liu ZW. 2018. Induction of P450 genes in Nilaparvata lugens and Sogatella furcifera by two neonicotinoid insecticides. Insect Sci 25:401-408. 10.1111/1744-7917.12440

Zeng D, Chen X, Peng J, Yang C, Peng M, Zhu W, Xie D, He P, Wei P, Lin Y, Zhao Y, and Chen X. 2018. Single-molecule

Peer) reviewing PDF | (2019:11:43416:3:0:NEW 6 May 2020) 
477

478

479

480

481

482

483

484

485

486

487

488 long-read sequencing facilitates shrimp transcriptome research. Sci Rep 8:16920. 10.1038/s41598-01835066-3

Zhang HM, Liu T, Liu CJ, Song S, Zhang X, Liu W, Jia H, Xue Y, and Guo AY. 2015. AnimalTFDB 2.0: a resource for expression, prediction and functional study of animal transcription factors. Nucleic Acids Res 43:D76-81. 10.1093/nar/gku887

Zhang Y, Yan Z, Qin Q, Nisenblat V, Chang HM, Yu Y, Wang T, Lu C, Yang M, Yang S, Yao Y, Zhu X, Xia X, Dang Y, Ren Y, Yuan P, Li R, Liu P, Guo H, Han J, He H, Zhang K, Wang Y, Wu Y, Li M, Qiao J, Yan J, and Yan L. 2018. Transcriptome Landscape of Human Folliculogenesis Reveals Oocyte and Granulosa Cell Interactions. Mol Cell. 10.1016/j.molcel.2018.10.029

Zhou G, Xu D, Xu D, and Zhang M. 2013. Southern rice black-streaked dwarf virus: a white-backed planthoppertransmitted fijivirus threatening rice production in Asia. Front Microbiol 4:270. 10.3389/fmicb.2013.00270 
Figure 1

(A) Distribution of the number and length of 12,514,449 subread sequences. (B) Distribution of the number and length of 388,348 FLNC sequences. (C) Distribution of the number and length of 251,109 consensus isoforms. 


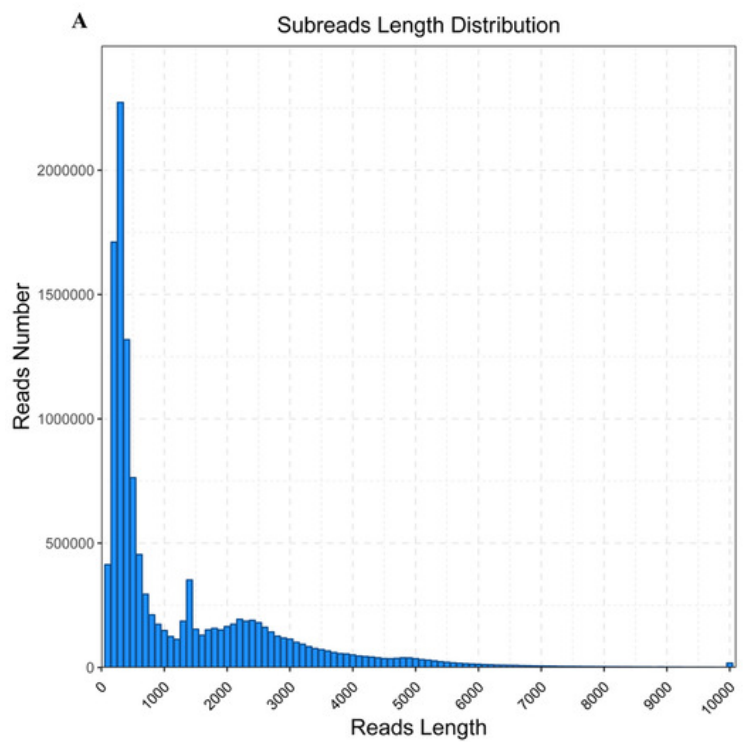

B

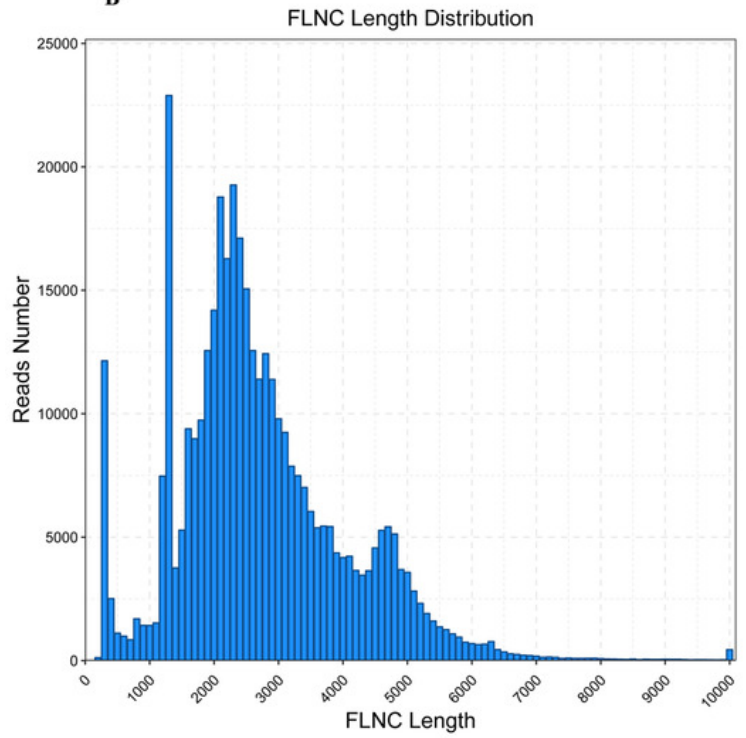

C

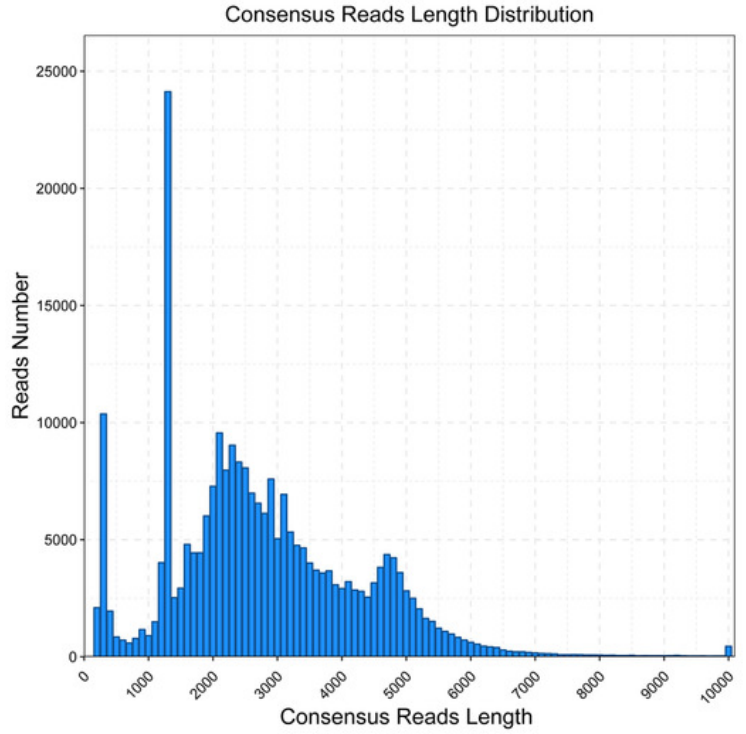

Peer) reviewing PDF | (2019:11:43416:3:0:NEW 6 May 2020) 
Figure 2

i) SSR motif unit: number of repeating bases. ii) "Mono-": repeat unit of a single base. iii) "Di": two bases. iv) "Tri-": three bases. v) "Tetra-": four bases. vi) "Penta-": five bases and "Hexa-": six bases. The specific number of repetitions should correspond to the legend according to the color.

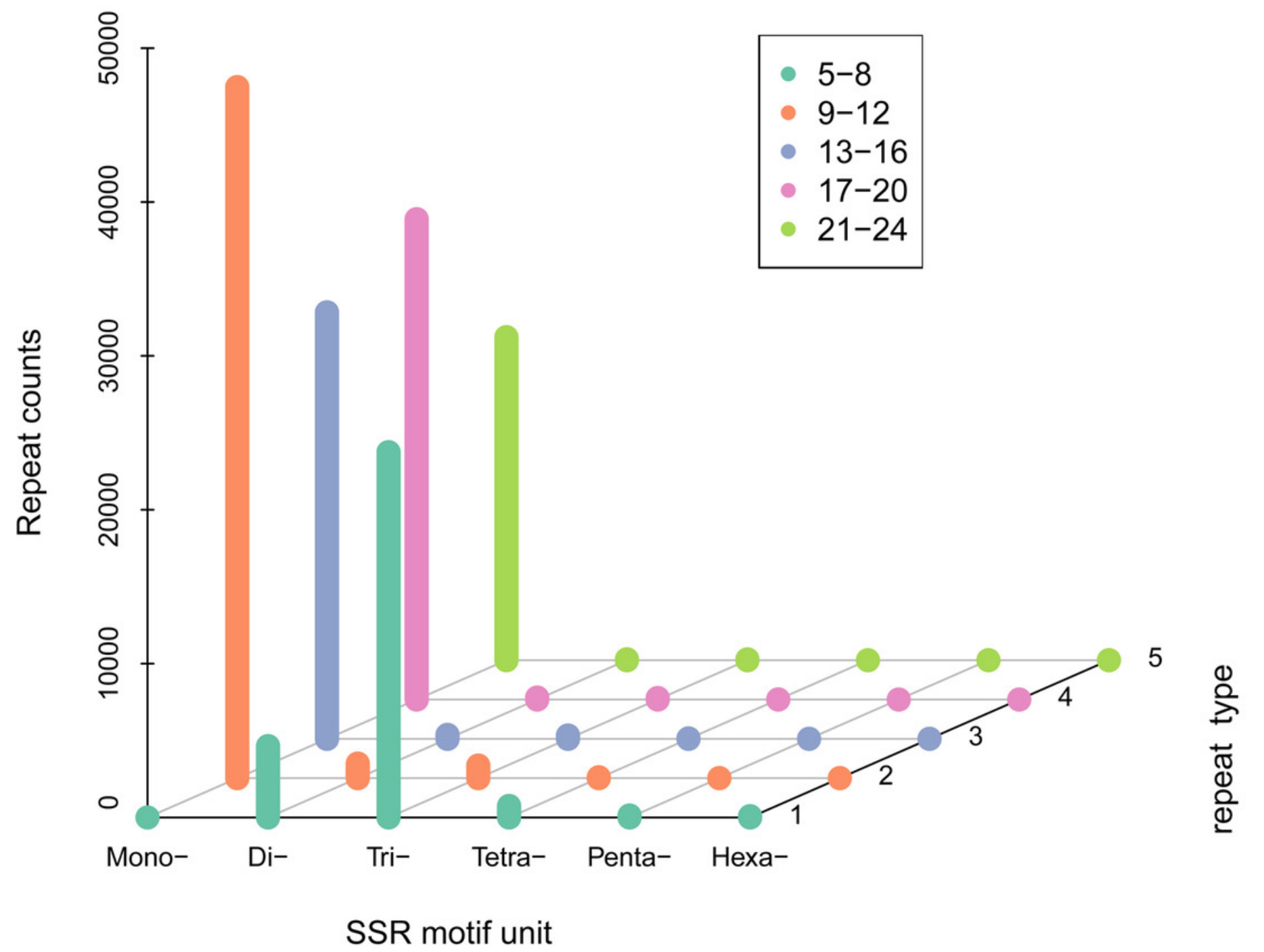


Figure 3

Different types of transcript family were plotted along the $x$-axis, while the number of transcription factors were plotted along the y-axis.

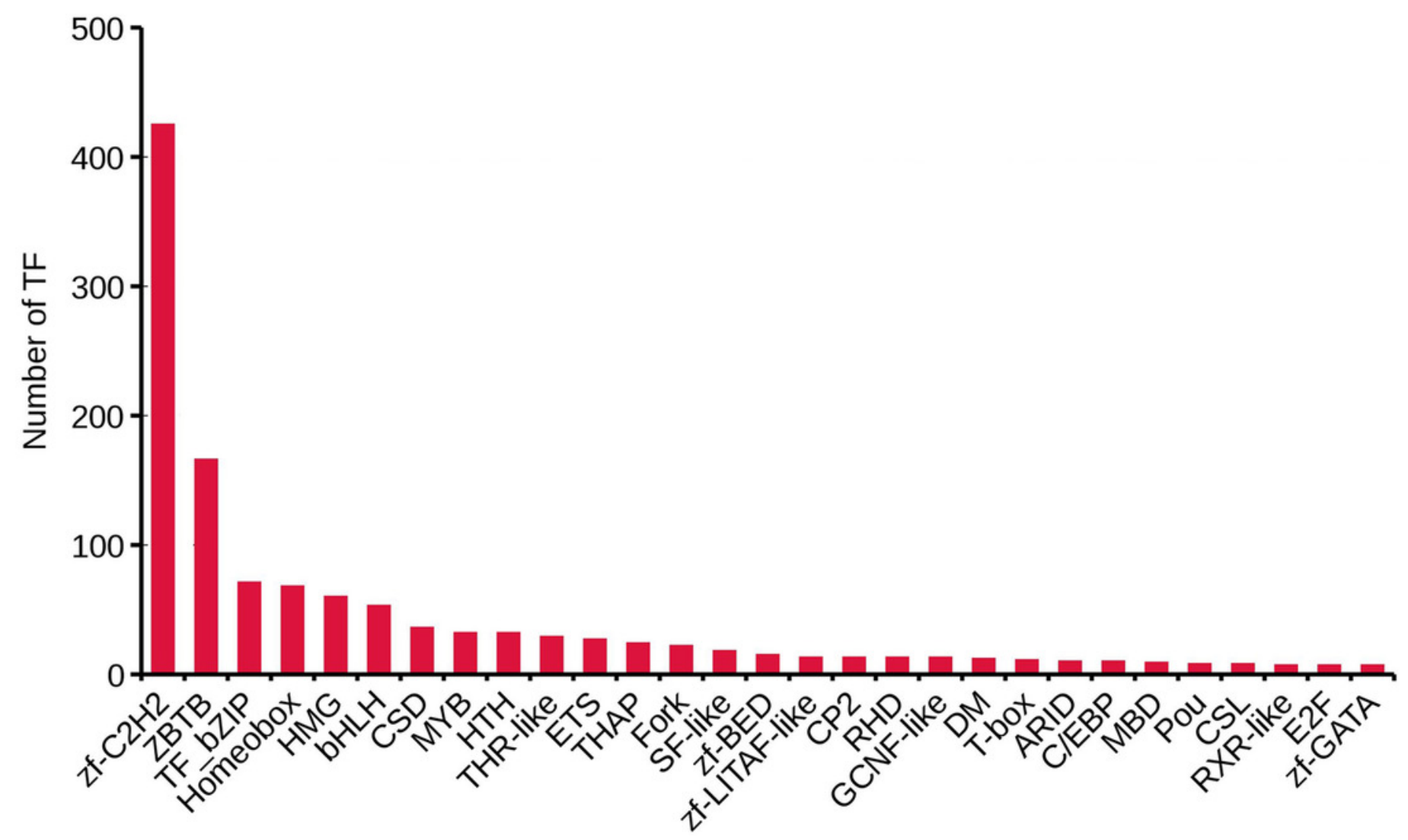

Transcript family 
Figure 4

Length of predicted CDS was plotted along the x-axis, while number of CDS transcripts was plotted along the left $y$-axis. The yellow line that represents the percentage of CDS length was plotted along the right $y$-axis.

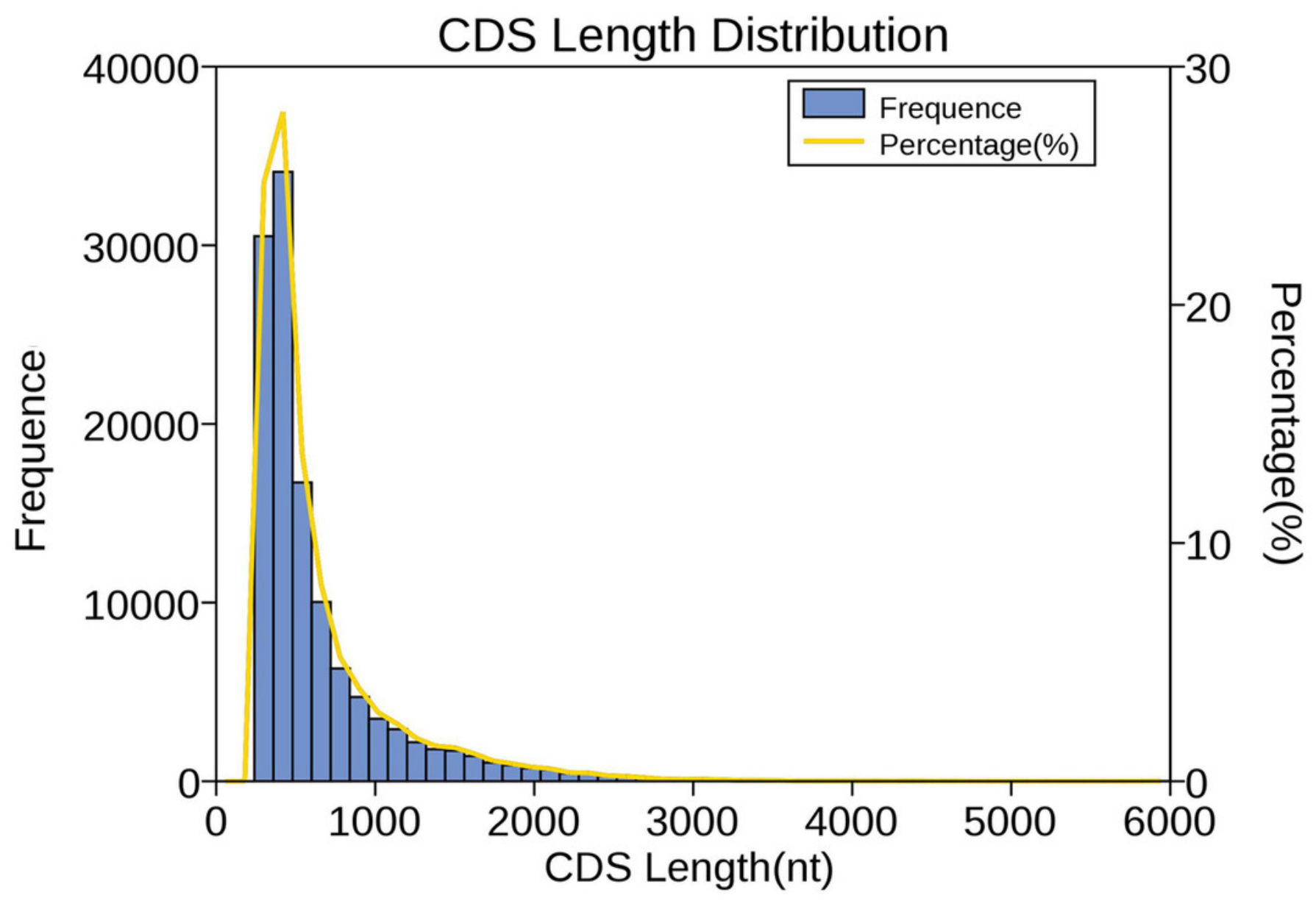




\section{Figure 5}

Non-overlapping areas indicate the number of IncRNAs identified by a single tool. Overlapping areas indicate the total number of IncRNAs identified by several tools.

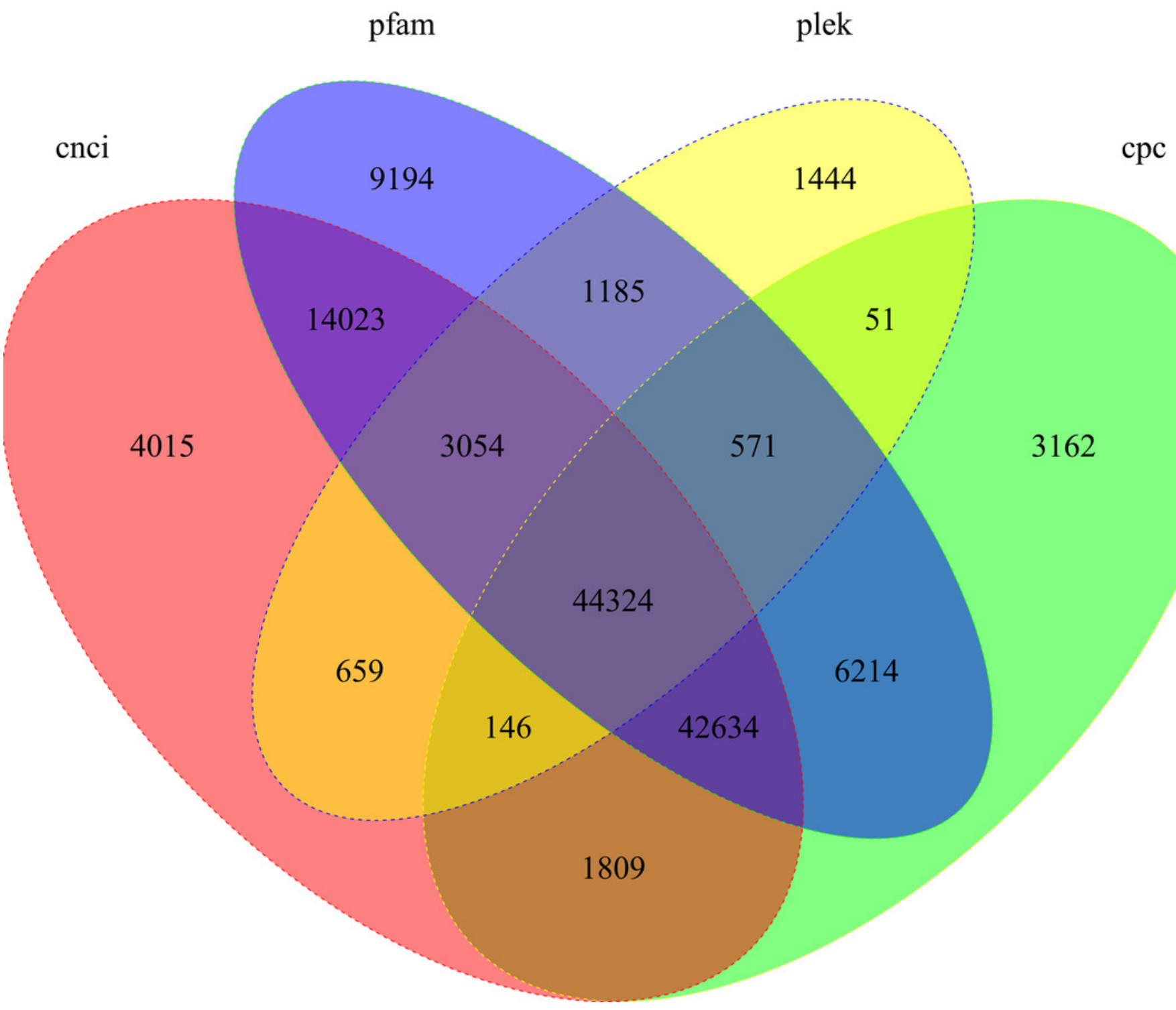




\section{Figure 6}

(A) Function annotation of transcripts in all databases. GO, Gene Ontology; KEGG, Kyoto Encyclopedia of Genes and Genomes; Nr, Non-Redundant Protein Database; COG, Cluster of Orthologous Groups of proteins. (B) Species of highest scoring blastp match in Nr. (C) KEGG pathway assignment of transcripts. (D) COG annotation of transcript sequences. (E) Distribution of GO terms for all annotated transcripts in cellular component, biological process and molecular function. 

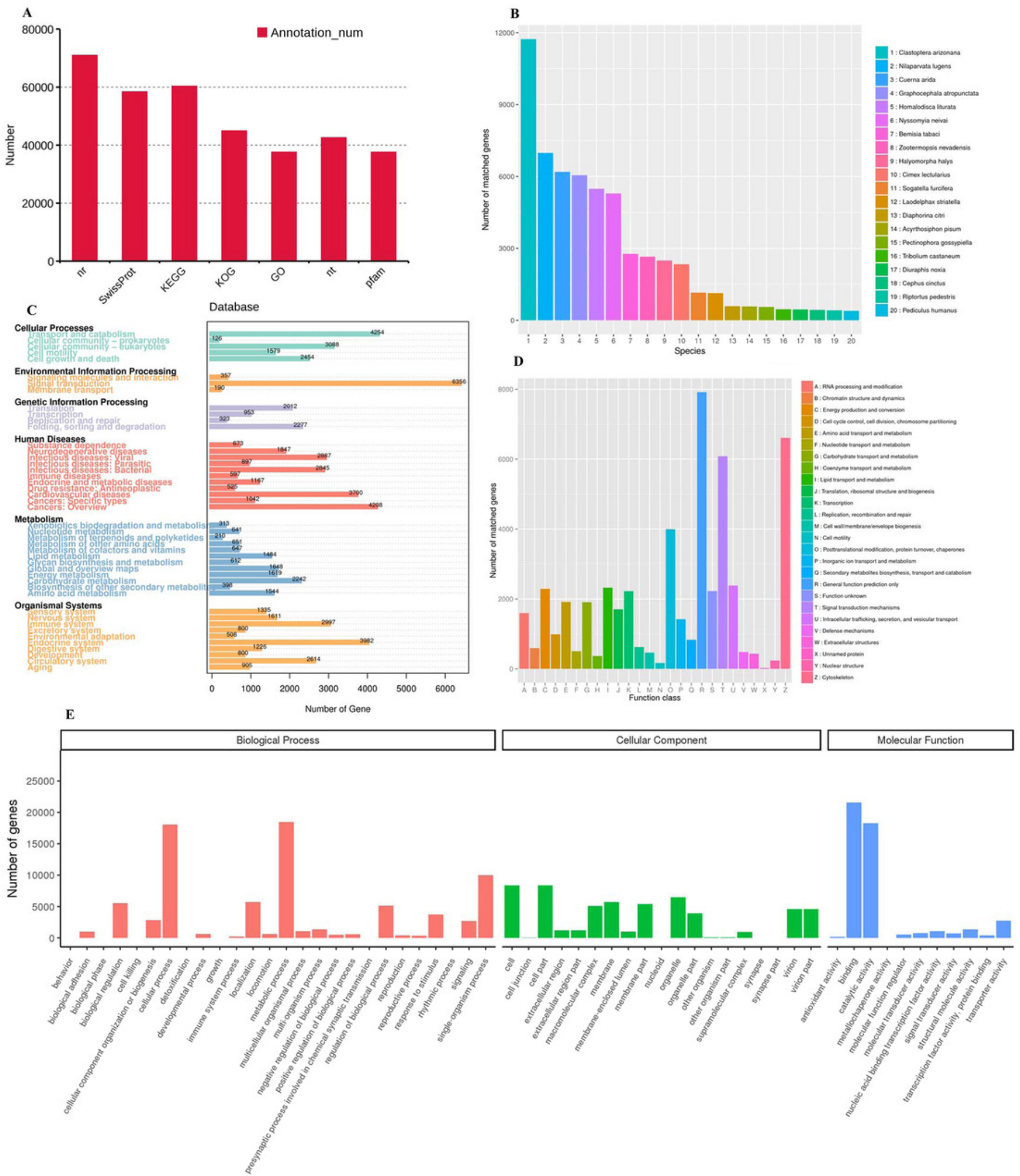


\section{Table $\mathbf{1}$ (on next page)}

The categories of genes are: i) complete BUSCOs: genes which match genes in the BUSCO reference group. ii) complete and single-copy BUSCOs: genes which match a single gene in the BUSCO reference group. iii) complete and duplicated BUSCOs: If these are found more than once they are classified as 'duplicated'. iv) fragmented BUSCOs: genes only partially recovered for which the gene length exceeds the alignment length cut-off. v) missing BUSCOs: not recovered genes. 
1

\begin{tabular}{llll}
\cline { 2 - 3 } 3 & BUSCO results & Count & Percentage (\%) \\
\cline { 2 - 4 } 4 & complete BUSCOs & 958 & $70.08 \%$ \\
5 & complete and single-copy BUSCOs & 605 & $44.26 \%$ \\
6 & complete and duplicated BUSCOs & 353 & $25.82 \%$ \\
7 & fragmented BUSCOs & 98 & $7.17 \%$ \\
8 & missing BUSCOs & 311 & $22.75 \%$ \\
9 & total & 1367 & \\
\cline { 2 - 3 }
\end{tabular}

10 


\section{Table 2 (on next page)}

i) Subread base: size of the valid insert subreads. ii) Subread number: number of valid insert subreads. iii) Average subread length: average length of subreads. vi) N50: represents the length of subreads that are more than $50 \%$ of the total length. 
1

\begin{tabular}{lllll}
\hline Sample & Subread bases $(\mathbf{G})$ & Subread number & Average subread length & N50 \\
\hline S. furcifera & 15.64 & $12,514,449$ & 1,250 & 2,665 \\
\hline
\end{tabular}

2 


\section{Table 3 (on next page)}

i) CCS: circular consensus sequence. ii) 5'-primer: number of reads with 5' signals. iii) 3'primer: number of reads with 3'signals. iv) Poly-A: number of reads with Poly-A tails. v) Full length: full-length reads. vi) FLNC: full-length non-chimeric reads. vii) Average FLNC read length: average length of full-length non-chimeric reads. viii) Consensus reads: number of reads of the non-redundant sequence obtained after clustering. 
1

\begin{tabular}{lllllllll}
\hline Sample & CCS & 5'-primer & 3'-primer & Poly-A & Full length & FLNC & $\begin{array}{l}\text { Average FLNC } \\
\text { read length }\end{array}$ & Consensus reads \\
\hline S. furcifera & 630,447 & 547,929 & 562,407 & 503,155 & 421,026 & 388,348 & 2,682 & 251,109 \\
\hline
\end{tabular}

2 


\section{Table 4 (on next page)}

Percent of transcripts or unigenes (\%): percentage of transcripts or unigenes in corresponding length range (bp). 
1

\begin{tabular}{lll}
\hline Length range (bp) & Transcripts & Unigenes \\
\hline$<500$ & $15253(6.1 \%)$ & $13966(8.9 \%)$ \\
$500-1000$ & $4139(1.7 \%)$ & $2304(1.5 \%)$ \\
$1000-2000$ & $62045(24.7 \%)$ & $31395(20.1 \%)$ \\
$2000-3000$ & $75229(30.0 \%)$ & $35699(22.9 \%)$ \\
$>3000$ & $94443(37.5 \%)$ & $72774(46.6 \%)$ \\
Total & 251109 & 156138 \\
\hline
\end{tabular}

2 


\section{Table 5 (on next page)}

1/2/3/4/5/6/7/8/9/10: number of genes containing the same number of transcripts. 
1

\begin{tabular}{lllllllllll}
\hline Isoform number & $\mathbf{1}$ & $\mathbf{2}$ & $\mathbf{3}$ & $\mathbf{4}$ & $\mathbf{5}$ & $\mathbf{6}$ & $\mathbf{7}$ & $\mathbf{8}$ & $\mathbf{9}$ & $\mathbf{1 0}$ \\
\hline Unigene number & 144,413 & 5,814 & 2,123 & 1,074 & 647 & 443 & 291 & 221 & 182 & 930 \\
\hline
\end{tabular}

2

3 